Article

\title{
Induced Action for Conformal Higher Spins from Worldline Path Integrals
}

\section{Roberto Bonezzi}

Physique Théorique et Mathématique, Université de Mons- UMONS 20, Place du Parc, 7000 Mons, Belgium; roberto.bonezzi@umons.ac.be

Received: 7 August 2017; Accepted: 29 August 2017; Published: 4 September 2017

Abstract: Conformal higher spin (CHS) fields, yet being non unitary, provide a remarkable example of a consistent interacting higher spin theory in flat space background, that is local to all orders. The non-linear action is defined as the logarithmically UV divergent part of a one-loop scalar effective action. In this paper we take a particle model, that describes the interaction of a scalar particle to the CHS background, and compute its path integral on the circle. We thus provide a worldline representation for the CHS action, and rederive its quadratic part. We plan to come back to the subject, to compute cubic and higher vertices, in a future work.

Keywords: higher spin fields; conformal symmetry; worldline formalism

\section{Introduction}

Four dimensional Maxwell $(s=1)$ theory is the first known example of a conformally invariant physical system. Similarly, massless matter lagrangians $(s=0,1 / 2)$ have conformal symmetry in flat space, that can be enhanced to general covariance plus local Weyl symmetry when coupled to a curved spacetime metric. On the other hand, for spin greater than one ordinary two-derivative theories, such as (super)gravities $(s=2,3 / 2)$ and massless higher spin theories $(s>2)$, are not conformal. Weyl squared gravity, with higher derivative lagrangian $\mathcal{L}=\sqrt{g}\left(W_{\mu v \rho \sigma}\right)^{2} \approx h^{\mu v} \square^{2} h_{\mu v}+\ldots$, and its supersymmetric extensions [1-3] are alternative models for $s \leq 2$ possessing local Weyl symmetry besides diffeomorphism invariance, and hence rigid conformal symmetry around flat space. Conformal higher spin fields (CHS) [3-14] are the $s>2$ generalization of the Weyl graviton and conformal gravitino. In four dimensional flat space they are described by the free lagrangian ${ }^{1}$

$$
S[h]=\sum_{s} \int d^{4} x h_{S} P_{s} \square^{s} h_{s},
$$

where $h_{s}=h_{\mu_{1} \ldots \mu_{s}}$ and $P_{s}$ is the spin-s transverse-traceless projector built out of $s$ powers of $P_{1}:=\delta_{v}^{\mu}-\frac{\partial^{\mu} \partial_{v}}{\square}$. The above action thus describes pure spin $s$ states (transverse and traceless) off-shell, and is invariant under differential and algebraic gauge transformations:

$$
\delta h_{s}=\partial \epsilon_{s-1}+\eta \alpha_{s-2}
$$

generalizing linearized diffeomorphisms and Weyl symmetry of conformal (super)gravity. The higher derivative $^{2}$ kinetic operator ensures locality of the action, at the price of formally loosing unitarity.

1 We will discuss only bosonic totally symmetric fields. In arbitrary even dimensions one has to add a power $\square^{\frac{d-4}{2}}$ of the laplacian.

2 One can describe CHS dynamics with ordinary two-derivative lagrangians, at the expense of introducing auxiliary fields $[15,16]$. 
Contrary to the case of free massless higher spins, that propagate only on maximally symmetric backgrounds, there is evidence [17-19] that conformal higher spins can propagate consistently on Bach-flat backgrounds, i.e., on the equations of motion of Weyl squared gravity. In fact, quite remarkably, the above action and linear gauge symmetry admit a consistent fully non-linear completion $[6,7,11]$, that is well defined around flat space and local to all orders in the fields. Indeed, unlike the case of massless higher spins, the absence of dimensionful parameters fixes the number of derivatives of each vertex uniquely. ${ }^{3}$ The CHS theory is power-counting renormalizable but, since Weyl and higher order algebraic symmetries are gauged, it has to be free of conformal and higher spin anomalies in order to be consistent at the quantum level. In the low spin case $s \leq 2$, vanishing of the total Weyl anomaly can be achieved only by $\mathcal{N}=4$ conformal supergravity coupled to four $\mathcal{N}=4$ SYM multiplets [20,21]. In the case of four dimensional CHS with one field of each integer spin, the a-coefficient ${ }^{4}$ of the Weyl anomaly vanishes upon a (regularized) summation over all spins [22,23], while cancellation of the c-coefficient [23-26], as well as the higher spin algebraic symmetries, seems less clear [19].

Besides being interesting on its own, as it gives a nontrivial example of an interacting higher spin theory in flat space, CHS fields are intimately related to massless higher spin theories in Anti de Sitter space [27-33] via the vectorial AdS/CFT correspondence [34-39]. Moreover, the non-linear CHS action naturally arises as an induced action $[6,7,11,40]$ in the holographic context: The free CFT of $N$ complex scalars admits an infinite number of conserved conformal currents of every spin in the $U(N)$ singlet sector, $J_{s} \sim \phi_{i}^{*} \partial^{s} \phi^{i}$. The dual fields to these conformal currents are identified with massless higher spin gauge fields in AdS space, whose boundary values $h_{s}$ source the $J_{s}$ currents and can in turn be seen as CHS fields on the boundary. The scalar path integral with sources $\sum_{s} J_{s} h_{s}$ yields the generating functional $\Gamma[h]$ of correlators of the conformal currents and, according to AdS/CFT correspondence, should be equal to the on-shell value of the, yet unknown ${ }^{5}$, action of massless higher spins in AdS 6 . However, the same generating functional $\Gamma[h]$ can be interpreted ${ }^{7}$, from a pure boundary viewpoint, as a one-loop effective action for the CHS fields $h_{s}$, that inherit the linearized gauge symmetry (2) thanks to conservation and tracelessness of the currents $J_{s}$. The logarithmically divergent part of $\Gamma[h]$ is local and gauge invariant and can be thus identified as the classical non-linear action $S_{\mathrm{CHS}}[h]$ for conformal higher spins [7,11].

The aim of this paper is to construct a quantum mechanical path integral to represent the effective action $\Gamma[h]$. Since the coupling $\sum_{s} J_{s} h_{s}$ is quadratic in the scalar fields, $\Gamma[h]$ is given by the functional determinant

$$
\Gamma[h]=N \log \operatorname{Det}[-\square+\hat{H}],
$$

3 In arbitrary even dimension $d$ the conformal weight of $h_{s}$ is $2-s$. Given an $n$th order vertex with fields of $\operatorname{spin}\left(s_{1}, \ldots, s_{n}\right)$, the number of derivatives is fixed to $N=d+\sum_{i=1}^{n} s_{i}-2 n$.

4 In four dimensions the Weyl anomaly contains only two relevant structures: the Euler density, whose coefficient is usually named $a$, and the square of the Weyl tensor, whose coefficient is $c$.

5 Vasiliev's equations lack a standard variational principle. Non-standard actions of covariant hamiltonian type have been proposed in [41-45]. From an holographic perspective, CFT correlators have been used to reconstruct AdS vertices in [46-48].

6 Direct matching of free gauge theory correlators with AdS Witten diagrams has been investigated in [49-51] in order to exploit open-closed string duality. In particular, in [49] one-loop open string diagrams in the field theory limit (hence worldline loops) were shown to reproduce tree level diagrams in AdS by direct change of variables in the moduli space.

7 In the standard AdS/CFT context [52-54] the boundary values of bulk fields are fixed, non dynamical sources for CFT correlators. From a pure boundary perspective, however, one can see the coupling $\sum_{s} J_{s} h_{s}$ as a Noether coupling that gauges the infinite dimensional symmetry algebra $[31,55]$ generated by the charges associated to the currents $J_{s}$. Moreover, even in the AdS/CFT context one can give different, Neumann type, boundary conditions to bulk fields, allowing them to fluctuate on the boundary $[22,56,57]$. 
where $\hat{H}$ is a differential operator linear in the CHS fields. Such type of one-loop effective actions is the most suitable to be computed by using first-quantized worldline models [58-69]. For instance, free massless scalar particles are described in first quantization by the relativistic worldline action

$$
S[x, p, e]=\int_{0}^{1} d \tau\left[p_{\mu} \dot{x}^{\mu}-\frac{e}{2} p^{2}\right] \Leftrightarrow S[x, e]=\int_{0}^{1} d \tau \frac{\dot{x}^{2}}{2 e},
$$

where $e(\tau)$ is the einbein, that enforces the mass-shell constraint $p^{2} \approx 0$ and, equivalently, ensures local $\tau$-reparametrization invariance. Coupling to a background curved metric $g_{\mu v}(x)$ and $U(1)$ gauge field $A_{\mu}(x)$ can be readily achieved by

$$
S_{g, A}[x, p, e]=\int_{0}^{1} d \tau\left[p_{\mu} \dot{x}^{\mu}-\frac{e}{2} g^{\mu v}\left(p_{\mu}-A_{\mu}\right)\left(p_{v}-A_{v}\right)\right] \Leftrightarrow S_{g, A}[x, e]=\int_{0}^{1} d \tau\left[\frac{1}{2 e} g_{\mu v} \dot{x}^{\mu} \dot{x}^{\nu}+A_{\mu} \dot{x}^{\mu}\right] .
$$

Quantization of the above actions on the circle gives the scalar loop contribution to the QFT one-loop effective action for gravitons and photons. To be precise, when the hamiltonian is not of the form $H=p^{2}+V(x)$, as it is the case in curved spacetime [70], the naive classical action does not give the correct quantum amplitudes, due to ordering issues in the quantum hamiltonian, and a local counterterm has to be added to the action (5) before using it in the path integral. One can add spinning degrees of freedom to the quantum particle [71-77], in order to give contributions of fields with nonzero spin in the loop. In the present case, since we are interested in the effective action $\Gamma[h]$ generated by a scalar loop, the scalar particle example will suffice.

In order to describe the interaction of the relativistic particle to background CHS fields, we shall employ the action proposed in [78], i.e.,

$$
S_{h}[x, p, e]=\int_{0}^{1} d \tau\left[p_{\mu} \dot{x}^{\mu}-e G(x, p)\right],
$$

where in the generalized hamiltonian $G(x, p)=p^{2}+\mathcal{H}(x, p)$ the conformal higher spin fields, contained in the $p$-power series expansion of $\mathcal{H}(x, p)$, are treated as perturbations over the flat space background $p^{2}$. In the low spin example (5) we gave the expression for the action both in phase space and configuration space. In most worldline applications one employs the configuration space action, but in the case at hand it seems much more convenient to stay with the phase space action and to perform the path integral directly in phase space. ${ }^{8}$ Indeed, the arbitrary dependence on momenta of $\mathcal{H}(x, p)$ makes the inversion $p=p(\dot{x})$ quite cumbersome, along with the appearence of inverse powers of $\dot{x}^{2}$ that would produce singularities in perturbation theory. The issue of quantum ordering of the operator $\hat{H}$ of (3) in relation to the classical interaction vertex $\mathcal{H}(x, p)$ will be discussed in the main text.

In the next section we start by reviewing the construction of $[7,11]$, that allows to find the explicit form of the operator $\hat{H}$. We proceed by introducing the above worldline model and discuss its symmetries. Finally, we quantize the action (6) by computing explicitly the path integral on the circle. By doing so we end up with a Scwhinger proper time representation of the effective action $\Gamma[h]$ that allows to extract the logarithmic divergence defining the classical action $S_{\mathrm{CHS}}[h]$. For illustrative purpose we shall rederive the quadratic action $[3,7,11]$, while we plan to address cubic and higher vertices in a future work. We conclude in Section 3 by pointing out some aspects of the present formalism that may be improved, and discussing some interesting directions for future investigations.

8 For very similar reasons, phase space worldline path integrals have been used in $[79,80]$ in the context of non-commutative field theory. 


\section{Induced Action for Conformal Higher Spins}

Let us start by considering a massless complex scalar field in flat spacetime of even dimension $d$, with action

$$
S_{0}[\phi]=\int d^{d} x \partial^{\mu} \phi^{*} \partial_{\mu} \phi .
$$

Being a free theory, it possesses an infinite number of conserved currents $J_{\mu(s)}=\phi^{*} \partial_{\mu_{1}} \ldots \partial_{\mu_{s}} \phi+\ldots 9$ of arbitrary integer spin $s=0,1,2, \ldots$ and conformal dimension $\Delta_{J_{s}}=d-2+s$, that can be made traceless thanks to conformal invariance [81,82]. Conservation $\partial^{v} J_{\nu \mu(s-1)} \approx 0$ and tracelessness $J^{\alpha}{ }_{\alpha \mu(s-2)} \approx 0$ hold on the scalar mass-shell $\square \phi \approx 0$. In this setting one can introduce conformal higher spin fields (CHS) via the Noether interactions

$$
S_{\text {int }}[\phi, h]=\sum_{s=0}^{\infty} \frac{(i)^{s}}{s !} \int d^{d} x J^{\mu(s)} h_{\mu(s)},
$$

that are invariant, on the free field equations $\square \phi \approx 0$, under the gauge transformations

$$
\delta_{\operatorname{lin}} h_{\mu(s)}=\partial_{\mu} \varepsilon_{\mu(s-1)}+\eta_{\mu \mu} \alpha_{\mu(s-2)}
$$

that are the linearized higher spin generalization of the gauge symmetries of conformal gravity. These on-shell symmetries can be deformed to full off-shell ones leaving invariant the total action

$$
S[\phi, h]=S_{0}[\phi]+S_{\text {int }}[\phi, h],
$$

by supplementing both the gauge fields and the scalar with extra transformations of the form

$$
\delta \phi=\mathcal{O}(\phi), \quad \delta h_{s}=\delta_{\operatorname{lin}} h_{s}+\mathcal{O}(h) .
$$

The UV logarithmically divergent part ${ }^{10}$ of the effective action $\Gamma[h]$, induced by the scalar path integral

$$
e^{-\Gamma[h]}=\int \mathcal{D} \phi^{*} \mathcal{D} \phi e^{-S[\phi, h]}=\operatorname{Det}^{-1}(-\square+\hat{H}),
$$

is local ${ }^{11}$ and invariant under the full transformation $\delta h_{s}=\partial \varepsilon_{s-1}+\eta \alpha_{s-2}+\mathcal{O}(h)$. It can thus be used to define a fully non-linear classical action for conformal higher spin fields, and at the quadratic level it has been shown to reproduce the free action of [3]. In (12) $\hat{H}$ is a differential operator linear in $h_{s}$, whose precise form will be now reviewed following [11].

\subsection{Noether Interaction and Symmetries}

The generating function of all the traceless conserved currents $J_{\mu(s)}$

$$
J(x, u):=\sum_{s=0}^{\infty} \frac{1}{s !} J_{\mu_{1} \ldots \mu_{s}}(x) u^{\mu_{1}} \ldots u^{\mu_{s}}=\sum_{s=0}^{\infty} J_{s}(x, u)
$$

obeying $\partial_{u} \cdot \partial_{x} J(x, u) \approx 0$ and $\partial_{u}^{2} J(x, u) \approx 0$ can be written as [11]

$$
J(x, u)=\Pi_{d} \mathcal{J}(x, u), \quad \mathcal{J}(x, u):=\phi^{*}(x+u / 2) \phi(x-u / 2),
$$

9 Indices denoted with the same letter and groups of indices $\mu(k)$ are intended as symmetrized with strength one, e.g., $J_{\mu(s)}:=J_{\left(\mu_{1} \ldots \mu_{s}\right)}$.

10 The logarithmic divergence is present only in even dimensions, that is the only case we will treat here.

11 The induced action contains vertices with arbitrary powers of higher spin fields but, due to the absence of dimensionful parameters, the number of derivatives is bounded by the number of fields and sum of the spins involved. 
where $\mathcal{J}(x, u)$ generates traceful conserved currents, that are mapped to the traceless ones by the operator

$$
\Pi_{d}:=\sum_{n=0}^{\infty} \frac{1}{n !\left(-\hat{N}-\frac{d-5}{2}\right)_{n}}\left[\frac{\partial^{2}-g \square}{16}\right]^{n},
$$

with the Pochhammer symbol defined by $(a)_{n}:=\frac{\Gamma(a+n)}{\Gamma(a)}$, and

$$
\hat{N}:=u \cdot \partial_{u}, \quad \partial:=u \cdot \partial_{x}, \quad g:=u^{2} .
$$

In terms of the higher spin generating function

$$
h(x, u):=\sum_{s=0}^{\infty} \frac{1}{s !} h_{\mu_{1} \ldots \mu_{s}}(x) u^{\mu_{1}} \ldots u^{\mu_{s}}=\sum_{s=0}^{\infty} h_{s}(x, u),
$$

the Noether interaction (8) can be written as

$$
\begin{aligned}
S_{\text {int }}[\phi, h] & =\left.\int d^{d} x J\left(x, i \partial_{u}\right) h(x, u)\right|_{u=0}=\left.\int d^{d} x \mathcal{J}\left(x, i \partial_{u}\right) \mathcal{H}(x, u)\right|_{u=0} \\
& =\left.\int d^{d} x e^{i \partial_{u} \cdot \partial_{v}} \mathcal{J}(x, v) \mathcal{H}(x, u)\right|_{u, v=0},
\end{aligned}
$$

where the transformed generating function of the gauge fields $\mathcal{H}(x, u)$ is obtained upon integrating by parts the spacetime derivatives in $\Pi_{d}$, and reads

$$
\mathcal{H}(x, u)=\mathcal{P}_{d} h(x, u), \quad \mathcal{P}_{d}:=\sum_{n=0}^{\infty} \frac{1}{n !\left(\hat{N}+n+\frac{d-3}{2}\right)_{n}}\left[\frac{\partial^{* 2}-\operatorname{Tr} \square}{16}\right]^{n},
$$

the inverse map being given by [11]

$$
h(x, u)=\mathcal{P}_{d}^{-1} \mathcal{H}(x, u), \quad \mathcal{P}_{d}^{-1}:=\sum_{n=0}^{\infty} \frac{(-1)^{n}}{n !\left(\hat{N}+\frac{d-1}{2}\right)_{n}}\left[\frac{\partial^{* 2}-\operatorname{Tr} \square}{16}\right]^{n},
$$

where we defined the divergence $\partial^{*}:=\partial_{u} \cdot \partial_{x}$ and trace $\operatorname{Tr}:=\partial_{u}^{2}$ operators. Despite the infinite series appearing in (19), each spin-s component of the conformal fields $h_{s}$ produces a finite tail of traces and divergences, as it can be seen by rewriting

$$
\mathcal{H}(x, u)=\sum_{s=0}^{\infty} \sum_{n=0}^{[s / 2]} \frac{1}{n !\left(s-n+\frac{d-3}{2}\right)_{n}}\left[\frac{\partial^{* 2}-\operatorname{Tr} \square}{16}\right]^{n} h_{s}(x, u) .
$$

By introducing the Fourier transform of $\mathcal{J}(x, v)$ in $v$-space:

$$
\mathcal{J}(x, v)=\int \frac{d^{d} p}{(2 \pi)^{d}} e^{-i v \cdot p} \rho(x, p)
$$

the interaction (18) can be further rewritten in the form

$$
S_{\text {int }}[\phi, h]=\int \frac{d^{d} x d^{d} p}{(2 \pi)^{d}} \rho(x, p) \mathcal{H}(x, p) .
$$

In [83] it has been shown that, upon introducing the first quantized Hilbert space where $x^{\mu}$ and $-i \frac{\partial}{\partial x^{\mu}}$ realize the algebra $\left[\hat{X}^{\mu}, \hat{P}_{v}\right]=i \delta_{v}^{\mu}$ and whereby the field $\phi(x)$ can be written as the wave 
function $\langle x \mid \phi\rangle$, the "density matrix" $\rho(x, p)$ is the Weyl symbol of the operator $|\phi\rangle\langle\phi|$. This allows, using the standard tools of Weyl quantization [84-86], to finally cast the action (23) as the inner product

$$
S_{\text {int }}[\phi, h]=\operatorname{Tr}[|\phi\rangle\langle\phi| \hat{H}]=\langle\phi|\hat{H}| \phi\rangle,
$$

where $\hat{H}(\hat{X}, \hat{P})$ is the operator with Weyl symbol given by $\mathcal{H}(x, p)$, i.e.,

$$
\hat{H}(\hat{X}, \hat{P})=\int \frac{d^{d} x d^{d} p}{(2 \pi)^{d}} \mathcal{H}(x, p) \int \frac{d^{d} y d^{d} k}{(2 \pi)^{d}} e^{i k \cdot(x-\hat{X})-i y \cdot(p-\hat{P})} .
$$

The total action entering the path integral (12) can thus be written as

$$
S[\phi, h]=\left\langle\phi\left|\hat{P}^{2}+\hat{H}(\hat{X}, \hat{P})\right| \phi\right\rangle,
$$

which is clearly invariant under

$$
|\phi\rangle \rightarrow \hat{O}^{-1}|\phi\rangle, \quad\left(\hat{P}^{2}+\hat{H}\right) \rightarrow \hat{O}^{\dagger}\left(\hat{P}^{2}+\hat{H}\right) \hat{O} .
$$

In terms of the hermitian operators $\hat{E}$ and $\hat{A}$ defined by $\hat{O}=\exp (\hat{A}+i \hat{E})$, the infinitesimal gauge transformations of the CHS fields contained in $\hat{H}$ are given by

$$
\delta \hat{H}=i\left[\hat{P}^{2}+\hat{H}, \hat{E}\right]+\left\{\hat{P}^{2}+\hat{H}, \hat{A}\right\}=i\left[\hat{P}^{2}, \hat{E}\right]+\left\{\hat{P}^{2}, \hat{A}\right\}+\mathcal{O}(h),
$$

the linearized transformations (9) descending from the action of the $\hat{P}^{2}$ part. The symmetries associated to $\hat{E}$ correspond to the differential ones $\delta_{\epsilon} h_{s}=\partial \epsilon_{s-1}+\ldots$ and are preserved at the quantum level, while the generator $\hat{A}$, corresponding to the generalized Weyl symmetry $\delta_{\alpha} h_{s}=\eta \alpha_{s-2}+\ldots$, develops a quantum anomaly due to the non invariant measure of the path integral (12). Nonetheless, it can be shown [11] that the UV logarithmically divergent part of the effective action preserves the full symmetry generated by $\hat{A}+i \hat{E}$, and can indeed be identified with the conformal higher spin action.

\subsection{Effective Action and Worldline Path Integral}

The effective action $\Gamma[h]$ is given by

$$
\Gamma[h]=\operatorname{Tr} \log \left(\hat{P}^{2}+\hat{H}\right)=-\int_{0}^{\infty} \frac{d T}{T} \operatorname{Tr}\left[e^{-T\left(\hat{P}^{2}+\hat{H}\right)}\right]
$$

in Schwinger proper time representation. The trace of the heat kernel

$$
K[T ; h]:=\operatorname{Tr}\left[e^{-T\left(\hat{P}^{2}+\hat{H}\right)}\right]
$$

admits a Laurent expansion in powers of $T$ when the higher spins in $\hat{H}$ are treated as a perturbation over the flat spin two background $\hat{P}^{2}$. Accordingly, upon introducing a cut-off $\Lambda$ in the small- $T$ region, the UV-regulated effective action can be organized according to its divergencies as

$$
\Gamma_{\Lambda}[h]:=-\int_{\frac{1}{\Lambda^{2}}}^{\infty} \frac{d T}{T} K[T ; h]=\sum_{n=1}^{\infty} \Lambda^{2 n} \Gamma_{n}[h]+\log \Lambda S_{\mathrm{CHS}}[h]+\Gamma_{\text {fin }}[h]+\mathcal{O}\left(\Lambda^{-2}\right),
$$

where the local, gauge invariant coefficient of the logarithmic divergence defines the conformal higher spin action being looked for.

As the heat kernel $K[T ; h]$ can be viewed as the trace of the (euclidean) evolution operator associated to the quantum mechanical Hamiltonian $\hat{P}^{2}+\hat{H}(\hat{X}, \hat{P})$, it is natural to represent it via a 
first quantized path integral. More so, the entire effective action (29) arises from the quantization of a relativistic particle model [78] that we briefly discuss. Consider the point particle hamiltonian action

$$
S[x, p ; e]=\int_{0}^{1} d \tau\left[p_{\mu} \dot{x}^{\mu}-e G(x, p)\right],
$$

where

$$
G(x, p)=p^{2}+\mathcal{H}(x, p) \approx 0
$$

is the generalized mass-shell constraint imposed by the Lagrange multiplier $e(\tau)$, that is associated with $\tau$-reparametrization invariance under

$$
\delta x^{\mu}=\xi\left\{x^{\mu}, G\right\}_{\text {P.B. }}, \quad \delta p_{\mu}=\xi\left\{p_{\mu}, G\right\}_{\text {P.B. }}, \quad \delta e=\dot{\xi},
$$

where $\{,\}_{\text {P.B. }}$ denotes the Poisson bracket and $\xi(\tau)$ is a worldline local parameter. The action (32) describes the propagation of a relativistic spinless particle in the background of the CHS fields contained in $\mathcal{H}$ according to (21). The Lagrange multiplier $e(\tau)$, called einbein, is the gauge field for $\tau$-reparametrizations, and it can be viewed as an intrinsic frame field on the worldline. The infinitesimal gauge transformations of the background fields, generated by

$$
\delta_{\epsilon} \mathcal{H}(x, p)=\left\{\epsilon(x, p), p^{2}+\mathcal{H}(x, p)\right\}_{\text {P.B. }},
$$

leave the action invariant when accompanied by the phase space transformations

$$
\delta_{\epsilon} x^{\mu}=\left\{x^{\mu}, \epsilon(x, p)\right\}_{\text {P.B. }, ~} \quad \delta_{\epsilon} p_{\mu}=\left\{p_{\mu}, \epsilon(x, p)\right\}_{\text {P.B. }} .
$$

This is the first quantized realization of the $\hat{E}$-type symmetries discussed in the field theory language, while the counterpart of the generalized Weyl symmetries $\hat{A}$ can be viewed as the invariance of the constraint surface $G(x, p) \approx 0$ under $G(x, p) \rightarrow e^{\alpha(x, p)} G(x, p)$. The action is indeed invariant under the combined transformations

$$
\delta_{\alpha} \mathcal{H}(x, p)=\alpha(x, p)\left(p^{2}+\mathcal{H}(x, p)\right), \quad \delta_{\alpha} e=-\alpha(x, p) e
$$

and, as we shall see next, the transformation of the einbein is responsible for breaking the $\alpha$-symmetry at the quantum level.

As it is well known from the cases of interaction with scalar, vector and gravitational backgrounds, the effective action $\Gamma[h]$ can be obtained by quantizing the action (32) on the circle:

$$
\Gamma[h]=\int_{S^{1}} \frac{D x D p D e}{\text { VolGauge }} e^{-S_{E}[x, p ; e]},
$$

where division by the gauge group volume entails the gauge fixing procedure for the local $\tau$-reparametrizations, and $S_{E}$ denotes the euclidean version of the action (32), i.e.,

$$
S_{E}[x, p ; e]=\int_{0}^{1} d \tau\left[-i p_{\mu} \dot{x}^{\mu}+e G(x, p)\right] .
$$

As mentioned in the Introduction, when the quantum hamiltonian contains mixing of coordinates and momenta, the naive path integral fails in general to provide the correct quantization. For a given classical hamiltonian $H(x, p)$, the functional integral in phase space produces transition amplitudes corresponding to the quantum Hamiltonian $\hat{H}_{W}(\hat{x}, \hat{p})$ obtained from the classical one by Weyl ordering [87]. The correctness of the choice of $\mathcal{H}$ as classical vertex is thus ensured by the relation (25), that greatly simplifies the model. In gauge fixing the local symmetry (34) on the circle, it is customary to fix the einbein to a constant: $e(\tau)=T$ that plays the role of Schwinger's proper 
time and breaks the Weyl symmetry (37). The ghost system associated to $\tau$-reparametrizations has locally trivial action, but its Faddeev-Popov determinant contributes on $S^{1}$ topology with a factor of $T^{-1}$, yielding

$$
\Gamma[h]=\int_{0}^{\infty} \frac{d T}{T} \int_{S^{1}} D x D p e^{-S_{E}[x, p ; T]},
$$

where the integral over $T$ is the finite dimensional remnant ${ }^{12}$ of the functional $e$-integral, and the gauge fixed action is simply obtained by replacing $e(\tau)=T$. We shall now compute the trace of the heat kernel

$$
K[T ; h]=\int_{S^{1}} D x D p e^{-S_{E}[x, p ; T]}
$$

by treating the phase space vertex $\mathcal{H}(x, p)$ as a perturbation over the free action

$$
S_{2}[x, p]=\int_{0}^{1} d \tau\left[T p^{2}-i p_{\mu} \dot{x}^{\mu}\right] .
$$

First of all we shall extract the zero mode from the periodic trajectories $x^{\mu}(\tau)$ :

$$
x^{\mu}(\tau)=x^{\mu}+q^{\mu}(\tau), \quad x^{\mu}:=\int_{0}^{1} d \tau x^{\mu}(\tau) \rightarrow \int_{0}^{1} d \tau q^{\mu}(\tau)=0,
$$

so that the functional measure splits as $\int_{S^{1}} D x=\int d^{d} x \int_{\mathrm{PBC}^{\prime}} D q$, where $\mathrm{PBC}^{\prime}$ denotes periodic boundary condition with the zero mode removed. Expectation values w.r.t. the free action (42) are denoted by

$$
\langle F(q, p)\rangle:=\frac{\int_{\mathrm{PBC}^{\prime}} D q \int D p F(q, p) e^{-S_{2}[q, p]}}{\int_{\mathrm{PBC}^{\prime}} D q \int D p e^{-S_{2}[q, p]}}
$$

and the trace of the heat kernel can be written as

$$
K[T ; h]=\int \frac{d^{d} x}{(4 \pi T)^{d / 2}}\left\langle e^{-T \int_{0}^{1} d \tau \mathcal{H}(x+q, p)}\right\rangle=\int \frac{d^{d} x}{(4 \pi T)^{d / 2}} \sum_{n=0}^{\infty} T^{n} \mathcal{V}_{n}[T ; h],
$$

where $(4 \pi T)^{-d / 2}$ is the value of the free path integral and the $n$-field effective vertex is given by

$$
\begin{aligned}
& \mathcal{V}_{n}[T ; h]=\frac{(-1)^{n}}{n !} \int_{0}^{1} d \tau_{1} \cdots \int_{0}^{1} d \tau_{n}\left\langle\prod_{i=1}^{n} \mathcal{H}\left(x+q\left(\tau_{i}\right), p\left(\tau_{i}\right)\right)\right\rangle \\
& =\frac{(-1)^{n}}{n !} \int_{0}^{1} d \tau_{1} \cdots \int_{0}^{1} d \tau_{n}\left\langle e^{\sum_{i=1}^{n} q_{i} \cdot \partial_{x_{i}}+p_{i} \cdot \partial_{u_{i}}}\right\rangle \mathcal{H}\left(x_{1}, u_{1}\right) \cdots \mathcal{H}\left(x_{n}, u_{n}\right) \begin{array}{l}
x_{i}=x \\
u_{i}=0
\end{array} \\
& =:\left.\hat{V}_{n}\left(T ; \partial_{x_{i}}, \partial_{u_{i}}\right) \mathcal{H}\left(x_{1}, u_{1}\right) \cdots \mathcal{H}\left(x_{n}, u_{n}\right)\right|_{\substack{x_{i}=x \\
u_{i}=0}}
\end{aligned}
$$

where $q_{i}:=q\left(\tau_{i}\right), p_{i}:=p\left(\tau_{i}\right)$ and we expanded the generating functions $\mathcal{H}\left(x+q_{i}, p_{i}\right)$ around $(x, 0)$. In terms of the currents

$$
j_{n}(\tau):=\sum_{i=1}^{n} \delta\left(\tau-\tau_{i}\right) \partial_{x_{i}}, \quad k_{n}(\tau):=\sum_{i=1}^{n} \delta\left(\tau-\tau_{i}\right) \partial_{u_{i}},
$$

the quantum average above can be recast in the form of a generating functional:

$$
\left\langle e^{\sum_{i=1}^{n} q_{i} \cdot \partial_{x_{i}}+p_{i} \cdot \partial_{u_{i}}}\right\rangle=\left\langle e^{\int_{0}^{1} d \tau\left[q(\tau) \cdot j_{n}(\tau)+p(\tau) \cdot k_{n}(\tau)\right]}\right\rangle
$$

12 The quantity $T=\int_{0}^{1} d \tau e(\tau)$ is gauge invariant on the circle; hence it constitutes a modulus to be integrated over after gauge fixing. 
This is a quadratic path integral and can be computed exactly, yielding

$$
\left\langle e^{\int_{0}^{1} d \tau\left[q(\tau) \cdot j_{n}(\tau)+p(\tau) \cdot k_{n}(\tau)\right]}\right\rangle=\exp \left\{\frac{1}{2} \int_{0}^{1} d \tau \int_{0}^{1} d \sigma K_{n}^{T}(\tau) G(\tau, \sigma) K_{n}(\sigma)\right\}
$$

for the column vector $K_{n}(\tau)=\left(\begin{array}{c}k_{n}(\tau) \\ j_{n}(\tau)\end{array}\right)$. Here $G(\tau, \sigma)$ is the matrix of the phase space Green's functions ${ }^{13}$

$$
\left\langle p_{\mu}(\tau) p_{v}(\sigma)\right\rangle=\frac{1}{2 T} \eta_{\mu v}, \quad\left\langle p_{\mu}(\tau) q^{v}(\sigma)\right\rangle=i \delta_{\mu}^{v} f(\sigma-\tau), \quad\left\langle q^{\mu}(\tau) q^{v}(\sigma)\right\rangle=2 T \eta^{\mu v} g(\tau-\sigma)
$$

with propagators

$$
f(\tau)=-\tau+\frac{1}{2} \operatorname{sign}(\tau), \quad g(\tau)=\frac{1}{2}\left(\tau^{2}-|\tau|+\frac{1}{6}\right), \quad \tau \in[-1,1] .
$$

By using the currents (47) in (49) one obtains

$$
\begin{aligned}
\hat{V}_{n}\left(T ; \partial_{x_{i}}, \partial_{u_{i}}\right) & =\frac{(-1)^{n}}{n !} \int_{0}^{1} d \tau_{1} \cdots \int_{0}^{1} d \tau_{n} \\
\times & \exp \frac{1}{2} \sum_{i, j=1}^{n}\left[\frac{1}{2 T} \partial_{u_{i}} \cdot \partial_{u_{j}}+2 i f\left(\tau_{i}-\tau_{j}\right) \partial_{x_{i}} \cdot \partial_{u_{j}}+2 T g\left(\tau_{i}-\tau_{j}\right) \partial_{x_{i}} \cdot \partial_{x_{j}}\right] .
\end{aligned}
$$

To manipulate it further, let us notice that $\sum_{i=1}^{n} \partial_{x_{i}} \sim 0$ is a total derivative, according to (46) and (45). This allows to consistently drop the constant part in every $g(\tau)$ propagator, leaving the effective propagator $\hat{g}(\tau):=\frac{1}{2}\left(\tau^{2}-|\tau|\right)$. The rigid translation invariance under $\tau_{i} \rightarrow \tau_{i}+c$, together with the periodicity of the trajectories over $S^{1}$, allows to fix one $\tau$ variable ${ }^{14}$, let us say $\tau_{n}=0$ and, thanks to the symmetry under permutations of the $\tau_{i}$, that is manifest from the first line of (46), one can also transform the $\tau$-integral: $\int_{0}^{1} d \tau_{1} \ldots \int_{0}^{1} d \tau_{n-1} \rightarrow(n-1) ! \int_{0}^{1} d \tau_{1} \int_{0}^{\tau_{1}} d \tau_{2} \ldots \int_{0}^{\tau_{n-2}} d \tau_{n-1}$, finally obtaining for the effective vertex

$$
\begin{aligned}
& \mathcal{V}_{n}[T ; h]=\frac{(-1)^{n}}{n} e^{\frac{1}{4 T}} \partial^{2} \int_{0}^{1} d \tau_{1} \int_{0}^{\tau_{1}} d \tau_{2} \cdots \int_{0}^{\tau_{n-2}} d \tau_{n-1} \\
& \times \exp \sum_{i<j}^{\left(\tau_{n}=0\right)}\left\{-i\left(\tau_{i j}-\frac{1}{2}\right)\left(\partial_{x_{i}} \cdot \partial_{u_{j}}-\partial_{x_{j}} \cdot \partial_{u_{i}}\right)+T \tau_{i j}\left(\tau_{i j}-1\right) \partial_{x_{i}} \cdot \partial_{x_{j}}\right\} \mathcal{H}\left(x_{1}, u_{1}\right) \cdots \mathcal{H}\left(x_{n}, u_{n}\right) \mid \begin{array}{l}
x_{i}=x \\
u_{i}=0
\end{array}
\end{aligned}
$$

where $\tau_{i j}:=\tau_{i}-\tau_{j}$ and $\partial_{U}:=\sum_{i=1}^{n} \partial_{u_{i}}$. For any given set of spins $\left\{s_{1}, \ldots, s_{n}\right\}$ of the CHS fields $h_{s_{i}}$, the maximal number of $u$-derivatives is bounded by $S:=\sum_{i=1}^{n} s_{i}$, so that the exponential $e^{\partial_{U}^{2} / 4 T}$ contributes with the maximal negative power $T^{-[S / 2]}$, making the Laurent expansion

$$
\mathcal{V}_{n}[T ; h]=\sum_{k=-\infty}^{\infty} T^{k} \mathcal{V}_{n}^{(k)}[h]
$$

13 See Appendix A for details.

14 This can be seen by just changing variables in integrals of periodic and translation invariant functions. However, a more precise justification comes from the gauge fixing procedure on the circle: The einbein $e(\tau)$ possesses, on $S^{1}$ topology, a Killing vector that is not fixed by the gauge $e(\tau)=T$ and that generates global translations around the circle. A natural way to fix the leftover global symmetry is then to fix the position of one vertex on the circle, e.g., by setting $\tau_{n}=0$, as it is customary in String Theory. 
well defined. The coefficient giving rise to the logarithmic divergence is thus the one of order $k=\frac{d}{2}-n$, and the CHS action can be identified as ${ }^{15}$

$$
S_{\mathrm{CHS}}[h]=\int d^{d} x \sum_{n=2}^{\infty} \mathcal{V}_{n}^{(d / 2-n)}[h] .
$$

All the vertices of (55) can be in principle computed using (53) but, since locality has to be manifest in the spin decomposition of the $h(x, u)$ basis, it would be desirable to develop a formalism that avoids the introduction of the $\mathcal{H}(x, u)$ generating function. In such a case, all the differential operators $\hat{V}_{n}\left(T ; \partial_{x_{i}}, \partial_{u_{i}}\right)$ should reduce to finite polynomials of homogeneous degree in spacetime derivatives.

\subsection{The Quadratic Action}

The effective vertex (53) is an equivalent representation of the one obtained in [11], but for illustrative purposes we shall rederive the quadratic action by computing $\mathcal{V}_{2}^{\left(\frac{d}{2}-2\right)}[h]$. From (53) one has

$$
\mathcal{V}_{2}[T ; h]=\left.\frac{1}{2} e^{\frac{1}{4 T} \partial_{U}^{2}+\frac{i}{2} \partial_{x} \cdot \partial u} \mathcal{F}\left(-i \partial_{x} \cdot \partial_{U}, T \square\right) \mathcal{H}(x, u) \mathcal{H}\left(x^{\prime}, u^{\prime}\right)\right|_{\substack{x^{\prime}=x \\ u, u^{\prime}=0}}
$$

where we used $\partial_{x^{\prime}} \sim-\partial_{x}$ and

$$
\mathcal{F}(\alpha, \beta):=\int_{0}^{1} d \tau e^{\alpha \tau+\beta \tau(1-\tau)}=\sum_{n=0}^{\infty} \frac{1}{n !} \mathcal{F}_{n}(\alpha) \beta^{n} .
$$

The functions $\mathcal{F}_{n}(\alpha)$ can be computed as hypergeometric integrals (A16):

$$
\mathcal{F}_{n}(\alpha)=\frac{(n !)^{2}}{(2 n+1) !}{ }_{1} F_{1}(n+1 ; 2 n+2 ; \alpha),
$$

and using Kummer's Formula (A17) can be recast in terms of Bessel functions (A9), giving

$$
\mathcal{V}_{2}[T ; h]=\left.\frac{\sqrt{\pi}}{2} e^{\frac{1}{4 T} \partial_{U}^{2}} \sum_{n=0}^{\infty}\left(\partial_{x} \cdot \partial_{U}\right)^{-n-\frac{1}{2}} J_{n+\frac{1}{2}}\left(\frac{\partial_{x} \cdot \partial_{U}}{2}\right)(T \square)^{n} \mathcal{H}(x, u) \mathcal{H}\left(x^{\prime}, u^{\prime}\right)\right|_{\substack{x^{\prime}=x \\ u, u^{\prime}=0}} .
$$

It is now possible to extract the contribution of order $T^{\frac{d}{2}-2}$, yielding

$$
\mathcal{V}_{2}^{\left(\frac{d-4}{2}\right)}[h]=\left.\sqrt{\frac{\pi}{8}}\left[\frac{1}{2} \sqrt{-\partial_{U \perp}^{2} \partial_{x}^{2}}\right]^{-\frac{d-3}{2}} J_{\frac{d-3}{2}}\left(\frac{1}{2} \sqrt{-\partial_{U \perp}^{2} \partial_{x}^{2}}\right)\left(\frac{\square}{2}\right)^{\frac{d-4}{2}} \mathcal{H}(x, u) \mathcal{H}\left(x^{\prime}, u^{\prime}\right)\right|_{\substack{x^{\prime}=x \\ u, u^{\prime}=0}},
$$

where we used Lommel's expansion Formula (A11), and the transverse projection is defined as

$$
v_{\perp}^{\mu}:=v^{\mu}-\frac{v \cdot \partial_{x} \partial_{x}^{\mu}}{\square}
$$

so that $\partial_{U \perp}^{2} \square=\partial_{U}^{2} \square-\left(\partial_{x} \cdot \partial_{U}\right)^{2}$. The above result coincides with the one of [11], and it can be seen that the Bessel function "undresses" the $\mathcal{H}$ fields, leaving a finite degree polynomial acting on the

15 The field independent $\mathcal{V}_{0}(T)=1$ cannot contribute to the logarithmic divergence and neither can the linear $\mathcal{V}_{1}[T ; h]$ in $d \geq 4$. 
two $h$ fields. To do so one applies Gegenbauer addition theorem (A13) to the above Bessel function, with the triplet of variables $Z:=\frac{1}{2} \sqrt{-\partial_{U \perp}^{2} \square}$ and ${ }^{16} z_{i}:=\frac{1}{2} \sqrt{-\partial_{u_{i} \perp}^{2} \square_{i}}$ obeying

$$
Z^{2}=z^{2}+z^{\prime 2}-2 z z^{\prime} w \text { for } w=\frac{\partial_{u \perp} \cdot \partial_{u^{\prime} \perp}}{\sqrt{\partial_{u \perp}^{2} \partial_{u^{\prime} \perp}^{2}}}
$$

The resulting $J_{n+\frac{d-3}{2}}\left(z_{i}\right)$ that appear from the addition theorem produce the inverse maps $\mathcal{P}_{d}^{-1}$ when acting on the corresponding $\mathcal{H}\left(x_{i}, u_{i}\right)$ as ${ }^{17}$

$$
\left.\left[z_{i}^{-n-\frac{d-3}{2}} J_{n+\frac{d-3}{2}}\left(z_{i}\right) \mathcal{H}\left(x_{i}, u_{i}\right)\right]\right|_{n}=h_{n}\left(x_{i}, u_{i}\right)
$$

and one is left with

$$
\mathcal{V}_{2}^{\left(\frac{d-4}{2}\right)}[h]=\left.\sqrt{\frac{\pi}{8}} \sum_{n=0}^{\infty} c_{n}(d)\left(\frac{\square}{2}\right)^{n+\frac{d-4}{2}}\left(\partial_{u \perp}^{2} \partial_{u^{\prime} \perp}^{2}\right)^{n / 2} C_{n}^{\frac{d-3}{2}}\left(\frac{\partial_{u \perp} \cdot \partial_{u^{\prime} \perp}}{\sqrt{\partial_{u \perp}^{2} \partial_{u^{\prime} \perp}^{2}}}\right) h(x, u) h\left(x^{\prime}, u^{\prime}\right)\right|_{\substack{x^{\prime}=x \\ u, u^{\prime}=0}},
$$

where $C_{n}^{v}(w)$ is the Gegenbauer polynomial and $c_{n}(d):=\frac{2^{-3 n-\frac{d-3}{2}}}{\Gamma\left(n+\frac{d-1}{2}\right)\left(\frac{d-3}{2}\right)_{n}}$. From the form of $\partial_{u_{i} \perp}$ one can see that the above expression is indeed local for each $n$ and of homogeneous degree $2 n+d-4$ in spacetime derivatives. It is also easy to view, from the definition of Gegenbauer polynomials (A14), that the sum over $n$ is diagonal in contractions, being of homogeneous degree $\left(\partial_{u} \partial_{u^{\prime}}\right)^{n}$. The above expression is proportional for each $n$ to the corresponding transverse and traceless projector ${ }^{18} P_{n}$, that can be displayed to write the quadratic action in the form (1) that is manifestly gauge invariant under the linearized transformations (9):

$$
S_{2 \mathrm{CHS}}=\int d^{d} x \sum_{s=0}^{\infty} c_{S} h_{s}(x, u) P_{S}\left(\overleftarrow{\partial_{u}}, \overrightarrow{\partial_{v}}\right) \square^{s+\frac{d-4}{2}} h_{s}(x, v)
$$

where we discarded a spin-independent constant and $c_{s}=\frac{1}{2^{3 s} \Gamma\left(s+\frac{d-1}{2}\right)}$.

\section{Discussion and Conclusions}

In this paper we have provided a worldline path integral representation for the non-linear conformal higher spin action $[7,11]$ in arbitrary even dimensions. We have rederived the quadratic part of the action, and we plan to come back in the future for the computation of cubic and higher vertices, some of which have been computed in [88,89], in transverse-traceless gauge, in the context of scattering amplitudes calculations.

The example of the quadratic action suggests that the "undressing" maps $\mathcal{P}_{d}^{-1}$ should appear at all orders in the differential operators $\hat{V}_{n}\left(T ; \partial_{x_{i}}, \partial_{u_{i}}\right)$, leaving finite degree polynomials acting on a string of fields $h_{s_{1}} \ldots h_{s_{n}}$. From the representation (53) it is not transparent how this should take place. For this reason, it would be interesting to find a way to avoid the introduction of the dressed generating function $\mathcal{H}(x, u)$, and work directly in the basis of CHS $h(x, u)$. To this goal, when restricting to four dimensions, a considerable advantage could come by working in terms of $\operatorname{sl}(2, \mathbb{C})$ spinors instead of tensors. All the trace projections would become trivial and one could work directly in terms of conformal primary currents $J_{s}$.

\footnotetext{
16 In the variables $z_{i}$ one can exchange $x$ with $x^{\prime}$ for free.

17 For details see the original derivation [11].

18 See appendix $C$ for the explicit form of the projectors.
} 
Another issue of (non-linear) field redefinitions is apparent when looking at the low spin content of the Noether interaction (8): the linear coupling

$$
\sum_{s=0}^{2} h_{s} J_{s} \sim h_{0} \phi^{*} \phi+h_{1}^{\mu} \phi^{*} \partial_{\mu} \phi+\frac{1}{2} h_{2}^{\mu v} \phi^{*} \partial_{\mu} \partial_{\nu} \phi
$$

does not coincide with the standard Weyl and $U(1)$ invariant coupling of a complex scalar to a vector gauge field in curved spacetime, i.e.,

$$
S=\int d^{d} x \sqrt{g}\left[g^{\mu \nu} D_{\mu} \phi^{*} D_{\nu} \phi+\frac{d-2}{4(d-1)} R \phi^{*} \phi\right]
$$

and the basis $\left(h_{0}, h_{1}, h_{2}\right)$ is related to the geometric $\left(A_{\mu}, g_{\mu v}=\eta_{\mu v}+h_{\mu \nu}\right)$ by a non-linear field redefinition. This issue has been discussed, for instance, in $[7,11,89]$. In fact, the covariant description of CHS fields in curved (maybe Bach-flat) background is still an open problem [17-19], and it underpins the question of vanishing Weyl anomalies. To this end, it would be interesting to find a first quantized origin of CHS fields, since at the worldline (or worldsheet) level it could be easier to achieve a covariant description, and the sum over spins, that is crucial in proving the vanishing of anomalies as well as triviality of scattering amplitudes, would be accounted for by the worldline fields.

Acknowledgments: We would like to thank Arkady Tseytlin, Evgeny Skvortsov, Dmitry Ponomarev and David De Filippi for useful discussions. The author thanks the Imperial College London and the Ludwig Maximilian University of Munich for kind hospitality during the final stages of this work. The work of R.B. was supported by a PDR "Gravity and extensions" from the F.R.S.-FNRS (Belgium).

Conflicts of Interest: The authors declare no conflict of interest.

\section{Appendix A. Worldline Phase Space Propagators}

In this section we will derive the phase space propagators associated with the euclidean worldline action on the circle

$$
S_{2}[x, p]=\int_{0}^{1} d \tau\left[T p^{2}-i p_{\mu} \dot{x}^{\mu}\right] .
$$

Upon extracting the zero mode from the periodic trajectories $x^{\mu}(\tau)$, one goes to Fourier space

$$
\begin{aligned}
& x^{\mu}(\tau)=x_{0}^{\mu}+q^{\mu}(\tau), \quad q^{\mu}(\tau)=\sum_{n \in \mathbb{Z} \backslash\{0\}} q_{n}^{\mu} e^{2 \pi i n \tau}, \\
& p_{\mu}(\tau)=\sum_{n \in \mathbb{Z}} p_{\mu n} e^{2 \pi i n \tau} .
\end{aligned}
$$

The Fourier modes obey the reality conditions

$$
\left(x_{0}, p_{0}\right) \in \mathbb{R}, \quad q_{n}^{*}=q_{-n}, \quad p_{n}^{*}=p_{-n}
$$

and the action (A1) reads

$$
S_{2}=T p_{0}^{2}+\sum_{n=1}^{\infty} Z_{n}^{\dagger} K_{n} Z_{n}
$$

in terms of the phase space vector $Z_{n}$ and kinetic matrix $K_{n}$

$$
Z_{n}:=\left(\begin{array}{l}
p_{n} \\
q_{n}
\end{array}\right), \quad K_{n}:=\left(\begin{array}{cc}
2 T & 2 \pi n \\
-2 \pi n & 0
\end{array}\right),
$$


where we suppressed all the spacetime indices. From the inverse matrix

$$
K_{n}^{-1}=\left(\begin{array}{cc}
0 & -\frac{1}{2 \pi n} \\
\frac{1}{2 \pi n} & \frac{2 T}{4 \pi^{2} n^{2}}
\end{array}\right)
$$

it is immediate to extract the two-point functions

$$
\left\langle p_{\mu}(\tau) p_{\nu}(\sigma)\right\rangle=\frac{\eta_{\mu \nu}}{2 T}, \quad\left\langle p_{\mu}(\tau) q^{\nu}(\sigma)\right\rangle=i \delta_{\mu}^{v} f(\sigma-\tau), \quad\left\langle q^{\mu}(\tau) q^{\nu}(\sigma)\right\rangle=2 T \eta_{\mu \nu} g(\tau-\sigma) .
$$

The above propagators are defined in terms of their Fourier series and read

$$
\begin{aligned}
& f(\tau):=\sum_{n=1}^{\infty} \frac{1}{\pi n} \sin (2 \pi n \tau)=-\tau+\frac{1}{2} \operatorname{sign}(\tau), \\
& g(\tau):=\sum_{n=1}^{\infty} \frac{1}{2 \pi^{2} n^{2}} \cos (2 \pi n \tau)=\frac{1}{2} \tau^{2}-\frac{1}{2}|\tau|+\frac{1}{12},
\end{aligned}
$$

where the latter expressions in terms of elementary functions hold in the interval $[-1,1]$.

\section{Appendix B. Special Functions}

We collect here the definitions and formulas that are relevant to the main text. The Bessel function of the first kind can be defined by the series

$$
J_{v}(z):=\sum_{k=0}^{\infty} \frac{(-1)^{k}}{k ! \Gamma(v+k+1)}\left(\frac{z}{2}\right)^{v+2 k},
$$

while the modified Bessel function $I_{v}(z)$ is given by

$$
I_{v}(z):=\sum_{k=0}^{\infty} \frac{1}{k ! \Gamma(v+k+1)}\left(\frac{z}{2}\right)^{v+2 k}=i^{-v} J_{v}(i z) .
$$

The Lommel expansion formula reads

$$
\sqrt{z+h}^{-v} J_{v}(\sqrt{z+h})=\sum_{k=0}^{\infty} \frac{1}{k !}\left(-\frac{h}{2}\right)^{k} \sqrt{z}^{-v-k} J_{v+k}(\sqrt{z})
$$

and, for a triplet $(\omega, x, y)$ obeying

$$
\omega^{2}=x^{2}+y^{2}-2 x y \cos \theta,
$$

one has the Gegenbauer addition theorem:

$$
\omega^{-v} J_{v}(\omega)=2^{v} \Gamma(v) \sum_{n=0}^{\infty}(v+n) x^{-v} J_{v+n}(x) y^{-v} J_{v+n}(y) C_{n}^{v}(\cos \theta),
$$

where $C_{n}^{v}(z)$ is the Gegenbauer polynomial defined by

$$
C_{n}^{v}(z)=\sum_{k=0}^{[n / 2]} \frac{(-1)^{k}(v)_{n-k}}{k !(n-2 k) !}(2 z)^{n-2 k} .
$$


The generalized hypergeometric function ${ }_{p} F_{q}$ is defined by the series

$$
{ }_{p} F_{q}\left(a_{1}, \ldots, a_{p} ; b_{1}, \ldots, b_{q} ; z\right)=\sum_{n=0}^{\infty} \frac{\left(a_{1}\right)_{n} \cdots\left(a_{p}\right)_{n}}{\left(b_{1}\right)_{n} \cdots\left(b_{q}\right)_{n}} \frac{z^{n}}{n !} .
$$

The confluent hypergeometric function ${ }_{1} F_{1}(a ; b ; z)$ admits the integral representation

$$
{ }_{1} F_{1}(a ; b ; z)=\frac{\Gamma(b)}{\Gamma(a) \Gamma(b-a)} \int_{0}^{1} d u e^{z u} u^{a-1}(1-u)^{b-a-1}
$$

and for $b=2 a$ it is related to the ${ }_{0} F_{1}$ series and thus to the Bessel function via Kummer's formula:

$$
{ }_{1} F_{1}(a ; 2 a ; z)=e^{z / 2}{ }_{0} F_{1}\left(; a+\frac{1}{2} ; \frac{z^{2}}{16}\right)=e^{z / 2}\left(\frac{z}{4}\right)^{\frac{1}{2}-a} \Gamma\left(a+\frac{1}{2}\right) I_{a-\frac{1}{2}}(z / 2) .
$$

\section{Appendix C. Transverse-Traceless Projectors}

The transverse-traceless projectors $P_{\mu(s)} v(s)$ of spin $s$, obeying

$$
\eta^{\alpha \beta} P_{\alpha \beta \mu(s-2)}{ }^{v(s)}=0=P_{\mu(s)}{ }^{\alpha \beta \nu(s-2)} \eta_{\alpha \beta}, \quad \partial^{\alpha} P_{\alpha \mu(s-1)^{v(s)}}=0=P_{\mu(s)}{ }^{\alpha v(s-1)} \partial_{\alpha}
$$

with normalization

$$
P_{\mu(s)}^{\lambda(s)} P_{\lambda(s)}{ }^{v(s)}=P_{\mu(s)}{ }^{v(s)},
$$

can be built from $s$ powers of the corresponding spin one transverse projector

$$
P_{\mu}^{v}:=\delta_{\mu}^{v}-\frac{\partial_{\mu} \partial^{v}}{\square}
$$

as

$$
P_{\mu(s)}{ }^{v(s)}=\sum_{k=0}^{[s / 2]} \alpha_{k}(s)\left(P_{\mu \mu} P^{v v}\right)^{k}\left(P_{\mu}{ }^{v}\right)^{s-2 k},
$$

with coefficients $\alpha_{k}(s)$ being fixed by tracelessness as

$$
\alpha_{k}(s)=\frac{(-1)^{k} s ! \Gamma\left(s-k+\frac{d-3}{2}\right)}{4^{k} k !(s-2 k) ! \Gamma\left(s+\frac{d-3}{2}\right)} .
$$

The generating function of the spin $s$ projector:

$$
P_{s}(u, v):=\frac{1}{s !} u^{\mu_{1}} \ldots u^{\mu_{s}} P_{\mu(s)}{ }^{v(s)} v_{v_{1}} \ldots v_{v_{s}}
$$

acts on the generating function of a spin $s$ field $h_{s}(x, u)=\frac{1}{s !} h_{\mu(s)}\left(u^{\mu}\right)^{s}$ as

$$
\left(P_{s} h_{s}\right)(x, u):=\frac{1}{s !} P_{\mu(s)}^{v(s)} h_{v(s)}(x)\left(u^{\mu}\right)^{s}=P_{s}\left(u, \partial_{v}\right) h_{s}(x, v),
$$

and it can be written in terms of Gegenbauer polynomials as

$$
P_{S}(u, v)=\frac{\Gamma\left(\frac{d-3}{2}\right)}{2^{s} \Gamma\left(s+\frac{d-3}{2}\right)}\left(\left|u_{\perp}\right|\left|v_{\perp}\right|\right)^{s} C_{s}^{\frac{d-3}{2}}\left(\frac{u_{\perp} \cdot v_{\perp}}{\left|u_{\perp}\right|\left|v_{\perp}\right|}\right),
$$

where transverse vectors are defined by

$$
u_{\perp}^{\mu}:=u^{\mu}-\frac{u \cdot \partial \partial^{\mu}}{\square} .
$$




\section{References}

1. Kaku, M.; Townsend, P.K.; van Nieuwenhuizen, P. Properties of Conformal Supergravity. Phys. Rev. D 1978, $17,3179-3187$.

2. Bergshoeff, E.; de Roo, M.; de Wit, B. Extended Conformal Supergravity. Nucl. Phys. B 1981, 182, $173-204$.

3. Fradkin, E.S.; Tseytlin, A.A. Conformal Supergravity. Phys. Rep. 1985, 119, 233-362.

4. Siegel, W. All Free Conformal Representations in All Dimensions. Int. J. Mod. Phys. A 1989, 4, 2015-2020.

5. Fradkin, E.S.; Linetsky, V.Y. Cubic Interaction in Conformal Theory of Integer Higher Spin Fields in Four-dimensional Space-time. Phys. Lett. B 1989, 231, 97-106.

6. Tseytlin, A.A. On limits of superstring in $A d S_{5} \times S^{5}$. Theor. Math. Phys. 2002, 133, 1376-1389.

7. Segal, A.Y. Conformal higher spin theory. Nucl. Phys. B 2003, 664, 59-130.

8. Shaynkman, O.V.; Tipunin, I.Y.; Vasiliev, M.A. Unfolded form of conformal equations in M dimensions and $\mathrm{o}(\mathrm{M}+2)$ modules. Rev. Math. Phys. 2006, 18, 823-886.

9. Marnelius, R. Lagrangian conformal higher spin theory. arXiv 2008, arXiv:0805.4686.

10. Vasiliev, M.A. Bosonic conformal higher-spin fields of any symmetry. Nucl. Phys. B 2010, 829, $176-224$.

11. Bekaert, X.; Joung, E.; Mourad, J. Effective action in a higher-spin background. J. High Energy Phys. 2011, $2011,48$.

12. Bandos, I.A.; de Azcarraga, J.A.; Meliveo, C. Extended supersymmetry in massless conformal higher spin theory. Nucl. Phys. B 2011, 853, 760-776.

13. Bekaert, X.; Grigoriev, M. Notes on the ambient approach to boundary values of AdS gauge fields. J. Phys. A 2013, 46, 214008.

14. Haehnel, P.; McLoughlin, T. Conformal Higher Spin Theory and Twistor Space Actions. arXiv 2016, arXiv:1604.08209.

15. Metsaev, R.R. Ordinary-derivative formulation of conformal low spin fields. J. High Energy Phys. 2012, 2012, 64.

16. Metsaev, R.R. Ordinary-derivative formulation of conformal totally symmetric arbitrary spin bosonic fields. J. High Energy Phys. 2012, 2012, 62.

17. Nutma, T.; Taronna, M. On conformal higher spin wave operators. J. High Energy Phys. 2014, $2014,66$.

18. Grigoriev, M.; Tseytlin, A.A. On conformal higher spins in curved background. J. Phys. A 2017, 50, 125401.

19. Beccaria, M.; Tseytlin, A.A. On induced action for conformal higher spins in curved background. Nucl. Phys. B 2017, 919, 359-383.

20. Fradkin, E.S.; Tseytlin, A.A. One Loop Beta Function in Conformal Supergravities. Nucl. Phys. B 1982, 203, 157-178.

21. Fradkin, E.S.; Tseytlin, A.A. Conformal Anomaly in Weyl Theory and Anomaly Free Superconformal Theories. Phys. Lett. B 1984, 134, 187-193.

22. Giombi, S.; Klebanov, I.R.; Pufu, S.S.; Safdi, B.R.; Tarnopolsky, G. AdS Description of Induced Higher-Spin Gauge Theory. J. High Energy Phys. 2013, 2013, 16.

23. Tseytlin, A.A. On partition function and Weyl anomaly of conformal higher spin fields. Nucl. Phys. B 2013, 877, 598-631.

24. Giombi, S.; Klebanov, I.R.; Safdi, B.R. Higher Spin $\mathrm{AdS}_{d+1} / \mathrm{CFT}_{d}$ at One Loop. Phys. Rev. D 2014, 89, 084004.

25. Beccaria, M.; Tseytlin, A.A. Higher spins in $\mathrm{AdS}_{5}$ at one loop: vacuum energy, boundary conformal anomalies and AdS/CFT. J. High Energy Phys. 2014, 2014, 114.

26. Beccaria, M.; Tseytlin, A.A. On higher spin partition functions. J. Phys. A 2015, 48, 275401.

27. Vasiliev, M.A. Consistent equation for interacting gauge fields of all spins in (3+1)-dimensions. Phys. Lett. $B$ 1990, 243, 378.

28. Vasiliev, M.A. Properties of equations of motion of interacting gauge fields of all spins in (3+1)-dimensions. Class. Quant. Grav. 1991, 8, 1387-1417.

29. Vasiliev, M.A. More on equations of motion for interacting massless fields of all spins in (3+1)-dimensions. Phys. Lett. B 1992, 285, 225-234.

30. Vasiliev, M.A. Higher spin gauge theories: Star product and AdS space. In The Many Faces of the Superworld; Shifman, M.A., Ed.; World Scientific: Singapore, 2000.

31. Vasiliev, M.A. Nonlinear equations for symmetric massless higher spin fields in (A)dS(d). Phys. Lett. B 2003, $567,139-151$. 
32. Bekaert, X.; Cnockaert, S.; Iazeolla, C.; Vasiliev, M.A. Nonlinear higher spin theories in various dimensions. arXiv 2005, arXiv:hep-th/0503128.

33. Didenko, V.E. Skvortsov, E.D. Elements of Vasiliev theory. arXiv 2014, arXiv:1401.2975.

34. Sezgin, E.; Sundell, P. Massless higher spins and holography. Nucl. Phys. B 2002, 644, 303-370; Erratum in 2003, 660, 403.

35. Klebanov, I.R.; Polyakov, A.M. AdS dual of the critical O(N) vector model. Phys. Lett. B 2002, 550, $213-219$.

36. Giombi, S.; Yin, X. Higher Spin Gauge Theory and Holography: The Three-Point Functions. J. High Energy Phys. 2010, 2010, 115.

37. Giombi, S.; Yin, X. Higher Spins in AdS and Twistorial Holography. J. High Energy Phys. 2011, $2011,86$.

38. Giombi, S.; Yin, X. The Higher Spin/Vector Model Duality. J. Phys. A 2013, 46, 214003.

39. Giombi, S. Higher Spin-CFT Duality. In New Frontiers in Fields and Strings, Proceedings of the 2015 Theoretical Advanced Study Institute in Elementary Particle Physics, Boulder, Colorado, 1-26 June 2015; World Scientific: Singapore, 2016; p. 137.

40. Liu, H.; Tseytlin, A.A. D $=4$ superYang-Mills, $\mathrm{D}=5$ gauged supergravity, and $\mathrm{D}=4$ conformal supergravity. Nucl. Phys. B 1998, 533, 88-108.

41. Boulanger, N.; Sundell, P. An action principle for Vasiliev's four-dimensional higher-spin gravity. J. Phys. A 2011, 44, 495402 .

42. Boulanger, N.; Colombo, N.; Sundell, P. A minimal BV action for Vasiliev's four-dimensional higher spin gravity. J. High Energy Phys. 2012, 2012, 43.

43. Boulanger, N.; Sezgin, E.; Sundell, P. 4D Higher Spin Gravity with Dynamical Two-Form as a Frobenius-Chern-Simons Gauge Theory. arXiv 2015, arXiv:1505.04957.

44. Bonezzi, R.; Boulanger, N.; Sezgin, E.; Sundell, P. An Action for Matter Coupled Higher Spin Gravity in Three Dimensions. J. High Energy Phys. 2016, 2016, 3.

45. Bonezzi, R.; Boulanger, N.; Sezgin, E.; Sundell, P. Frobenius-Chern-Simons gauge theory. J. Phys. A 2017, 50,055401

46. Bekaert, X.; Erdmenger, J.; Ponomarev, D.; Sleight, C. Towards holographic higher-spin interactions: Four-point functions and higher-spin exchange. J. High Energy Phys. 2015, 2015, 170.

47. Bekaert, X.; Erdmenger, J.; Ponomarev, D.; Sleight, C. Quartic AdS Interactions in Higher-Spin Gravity from Conformal Field Theory. J. High Energy Phys. 2015, 2015, 149.

48. Sleight, C.; Taronna, M. Higher Spin Interactions from Conformal Field Theory: The Complete Cubic Couplings. Phys. Rev. Lett. 2016, 116, 181602.

49. Gopakumar, R. From free fields to AdS. Phys. Rev. D 2004, 70, 025009.

50. Gopakumar, R. From free fields to AdS. 2. Phys. Rev. D 2004, 70, 025010.

51. Gopakumar, R. From free fields to AdS: III. Phys. Rev. D 2005, 72, 066008.

52. Maldacena, J.M. The Large N limit of superconformal field theories and supergravity. Int. J. Theor. Phys. 1999, $38,1113-1133$.

53. Gubser, S.S.; Klebanov, I.R.; Polyakov, A.M. Gauge theory correlators from noncritical string theory. Phys. Lett. B 1998, 428, 105-114.

54. Witten, E. Anti-de Sitter space and holography. Adv. Theor. Math. Phys. 1998, 2, 253-291.

55. Eastwood, M.G. Higher symmetries of the Laplacian. Ann. Math. 2005, 161, 1645-1665.

56. Balasubramanian, V.; Gimon, E.G.; Minic, D.; Rahmfeld, J. Four-dimensional conformal supergravity from AdS space. Phys. Rev. D 2001, 63, 104009.

57. Compere, G.; Marolf, D. Setting the boundary free in AdS/CFT. Class. Quant. Grav. 2008, 25, 195014.

58. Schubert, C. Perturbative quantum field theory in the string inspired formalism. Phys. Rept. 2001, 355, 73-234.

59. Bern, Z.; Kosower, D.A. The Computation of loop amplitudes in gauge theories. Nucl. Phys. B 1992, 379, 451-561.

60. Strassler, M.J. Field theory without Feynman diagrams: One loop effective actions. Nucl. Phys. B 1992, 385, 145-184.

61. Bastianelli, F.; van Nieuwenhuizen, P. Trace anomalies from quantum mechanics. Nucl. Phys. B 1993, 389, 53-80.

62. D’Hoker, E.; Gagne, D.G. Worldline path integrals for fermions with general couplings. Nucl. Phys. B 1996, 467, 297-312. 
63. Reuter, M.; Schmidt, M.G.; Schubert, C. Constant external fields in gauge theory and the spin $0,1 / 2,1$ path integrals. Ann. Phys. 1997, 259, 313-365.

64. Bastianelli, F.; Zirotti, A. Worldline formalism in a gravitational background. Nucl. Phys. B 2002, 642, 372-388.

65. Bastianelli, F.; Corradini, O.; Zirotti, A. Dimensional regularization for $\mathrm{N}=1$ supersymmetric sigma models and the worldline formalism. Phys. Rev. D 2003, 67, 104009.

66. Bastianelli, F.; Benincasa, P.; Giombi, S. Worldline approach to vector and antisymmetric tensor fields. J. High Energy Phys. 2005, 2005, 10.

67. Dai, P.; Huang, Y.T.; Siegel, W. Worldgraph Approach to Yang-Mills Amplitudes from N=2 Spinning Particle. J. High Energy Phys. 2008, 2008, 27.

68. Bastianelli, F.; Bonezzi, R. One-loop quantum gravity from a worldline viewpoint. J. High Energy Phys. 2013, 2013, 16.

69. Bastianelli, F.; Bonezzi, R.; Corradini, O.; Latini, E. Particles with non abelian charges. J. High Energy Phys. 2013, 2013, 98.

70. Bastianelli, F.; van Nieuwenhuizen, P. Path Integrals and Anomalies in Curved Space; Cambridge University Press: Cambridge, UK, 2006.

71. Gershun, V.D.; Tkach, V.I. Classical And Quantum Dynamics Of Particles With Arbitrary Spin. J. Exp. Theor. Phys. Lett. 1979, 29, 288-291.

72. Henneaux, M.; Teitelboim, C. First and second quantized point particles of any spin. In Quantum Mechanics of Fundamental Systems 2; Springer: Berlin, Germany, 1989; pp. 113-152.

73. Howe, P.S.; Penati, S.; Pernici, M.; Townsend, P.K. Wave Equations for Arbitrary Spin From Quantization of the Extended Supersymmetric Spinning Particle. Phys. Lett. B 1988, 215, 555-558.

74. Kuzenko, S.M.; Yarevskaya, Z.V. Conformal invariance, N extended supersymmetry and massless spinning particles in anti-de Sitter space. Mod. Phys. Lett. A 1996, 11, 1653-1664.

75. Bastianelli, F.; Corradini, O.; Latini, E. Spinning particles and higher spin fields on (A)dS backgrounds. J. High Energy Phys. 2008, 2008, 54.

76. Bastianelli, F.; Corradini, O.; Waldron, A. Detours and Paths: BRST Complexes and Worldline Formalism. J. High Energy Phys. 2009, 2009, 17.

77. Bastianelli, F.; Bonezzi, R.; Corradini, O.; Latini, E. Effective action for higher spin fields on (A)dS backgrounds. J. High Energy Phys. 2012, 2012, 113.

78. Segal, A.Y. Point particle in general background fields versus gauge theories of traceless symmetric tensors. Int. J. Mod. Phys. A 2003, 18, 4999-5021.

79. Bonezzi, R.; Corradini, O.; Franchino Vinas, S.A.; Pisani, P.A.G. Worldline approach to noncommutative field theory. J. Phys. A 2012, 45, 405401.

80. Ahmadiniaz, N.; Corradini, O.; D’Ascanio, D.; Estrada-Jiménez, S.; Pisani, P. Noncommutative U(1) gauge theory from a worldline perspective. J. High Energy Phys. 2015, 2015, 69.

81. Craigie, N.S.; Dobrev, V.K.; Todorov, I.T. Conformally Covariant Composite Operators in Quantum Chromodynamics. Ann. Phys. 1985, 159, 411-444.

82. Berends, F.A.; Burgers, G.J.H.; van Dam, H. Explicit Construction of Conserved Currents for Massless Fields of Arbitrary Spin. Nucl. Phys. B 1986, 271, 429-441.

83. Bekaert, X.; Joung, E.; Mourad, J. On higher spin interactions with matter. J. High Energy Phys. 2009, $2009,126$.

84. Weyl, H. Quantum mechanics and group theory. Z. Phys. 1927, 46, 1.

85. Wigner, E.P. On the quantum correction for thermodynamic equilibrium. Phys. Rev. 1932, 40, 749-759.

86. Moyal, J.E. Quantum mechanics as a statistical theory. Proc. Camb. Philos. Soc. 1949, 45, 99-124.

87. Sato, M.A. Operator Ordering and Perturbation Expansion in the Path Integration Formalism. Prog. Theor. Phys. 1977, 58, 1262-1270.

88. Joung, E.; Nakach, S.; Tseytlin, A.A. Scalar scattering via conformal higher spin exchange. J. High Energy Phys. 2016, 2016, 125.

89. Beccaria, M.; Nakach, S. Tseytlin, A.A. On triviality of S-matrix in conformal higher spin theory. J. High Energy Phys. 2016, 2016, 34.

(C) 2017 by the author. Licensee MDPI, Basel, Switzerland. This article is an open access article distributed under the terms and conditions of the Creative Commons Attribution (CC BY) license (http:/ / creativecommons.org/licenses/by/4.0/). 